\title{
The impact of riociguat on clinical parameters and quality of life in patients with chronic thromboembolic pulmonary hypertension - results of a retrospective clinical registry
}

\author{
Pavel Jansa ${ }^{a}$, David Ambroz ${ }^{\mathrm{a}}$, Jan Kuchar ${ }^{\mathrm{b}}$, Vladimir Dytrych ${ }^{\mathrm{a}}$, Jaroslav Lindnerc, Ales Linhart ${ }^{\mathrm{a}}$
}

\begin{abstract}
Aims. The primary objective of the registry was to assess the impact of riociguat on clinical parameters and quality of life in patients with chronic thromboembolic pulmonary hypertension (CTEPH) that was inoperable or persistent/recurrent after pulmonary endarterectomy (PEA). In contrast to randomized pivotal trials, this non-interventional registry evaluated the effectiveness and safety of riociguat in a real-world setting.

Methods. Retrospective data were collected from patients' charts as recorded in routine clinical practice from the initiation of riociguat therapy up to approximately 5 months and 1 year after this initiation.

Results. In total, 51 patients from a single site were enrolled. After 5 months (mean duration) of riociguat treatment, the following improvements from baseline were observed: change of distance in the 6-minute walking distance (6MWD) $(P=0.066)$; change of score from the quality of life questionnaire (EQ5D-5L) $(P=0.020)$, and overall self-assessment of health status $(P=0.001)$. New York Heart Association (NYHA) class improved in $24.3 \%$ of patients. After 11.2 months (mean duration) of riociguat treatment, the following improvements from baseline were observed: change of distance in the 6MWD test $(P=0.006)$, and overall self-assessment of health status $(P=0.009)$. NYHA class improved in $46.4 \%$ of patients. Riociguat was well tolerated. In total, 4 patients reported side effects, with hospitalization required in one case and 2 patients who had to discontinue the treatment. Annual survival rate was $89.1 \%$.

Conclusion. Riociguat improves functional NYHA class, distance in the 6MWD test and quality of life in a real-world patient population.
\end{abstract}

Key words: riociguat, chronic thromboembolic pulmonary hypertension, clinical parameters, quality of life, real-world population

Received: November 18, 2019; Revised: December 13, 2019; Accepted: December 13, 2019; Available online: January 20, 2020 https://doi.org/10.5507/bp.2019.061

(c) 2021 The Authors; https://creativecommons.org/licenses/by/4.0/

${ }^{a}{ }^{\text {nd }}$ Department of Medicine - Department of Cardiovascular Medicine, First Faculty of Medicine, Charles University and General University Hospital in Prague, Czech Republic

${ }^{b}$ Department of Cardiology, Tabor Hospital, Inc., Czech Republic

${ }^{c}{ }^{\text {nd }}$ Department of Surgery - Department of Cardiovascular Surgery, First Faculty of Medicine, Charles University and General University Hospital in Prague, Czech Republic

Corresponding author: Pavel Jansa, e-mail: pavel.jansa@vfn.cz

\section{INTRODUCTION}

Chronic thromboembolic pulmonary hypertension (CTEPH) is a chronic complication of acute pulmonary embolism, where persistent thrombotic obstructions formed in pulmonary vessels and concurrent peripheral vascular remodelling with abnormal angiogenesis, disordered fibrinolysis and endothelial dysfunction cause precapillary pulmonary hypertension and increased right ventricular strain, leading to right heart failure and premature death ${ }^{1}$. CTEPH develops in approximately $2-4 \%$ of patients who survive acute pulmonary embolism ${ }^{2}$.

Surgical pulmonary endarterectomy (PEA) is the treatment modality that is indicated for $60-70 \%$ of patients with confirmed CTEPH. The remaining patients are considered inoperable, most frequently due to surgically inaccessible lesions or due to comorbidities. After
PEA, up to $20-30 \%$ of patients develop persistent/recurrent CTEPH (ref. $\left.{ }^{3}\right)$.

Patients with persistent/recurrent CTEPH and patients who are considered inoperable for peripheral involvement have the option of either undergoing balloon pulmonary angioplasty (BPA) and/or specific pharmacotherapy with the only commercially marketed drug called riociguat. Riociguat is an oral stimulator of soluble guanylate cyclase $(\mathrm{sGC})$ that increases the sensitivity of sGC to nitric oxide (NO), resulting in increased cyclic guanosine monophosphate (cGMP) levels ${ }^{4,5}$. Riociguat also elevates cGMP production independently of NO. Riociguat efficacy and safety in CTEPH has been demonstrated in the randomized clinical trial CHEST-1 (Chronic Thromboembolic Pulmonary Hypertension sGC-Stimulator Trial) with a population of 261 patients with inoperable CTEPH or residual pulmonary hypertension after PEA $\left(\right.$ ref. $\left.^{6}\right)$. 
Here we present the results of a registry collecting retrospective data of patients with inoperable CTEPH or persistent/recurrent CTEPH receiving riociguat therapy in a real-world setting.

\section{METHODS}

Retrospective data was collected from patients with CTEPH diagnosed and treated at the Pulmonary Hypertension Centre in General University Hospital in Prague, Czech Republic. Data collection was conducted in accordance with the principles laid down in the 18th World Medical Assembly (Helsinki, 1964), including all subsequent amendments, and in compliance with all laws and regulations of the Czech Republic. The approval of retrospective data collection was provided by the ethics committee of the General University Hospital in Prague (ID 1208/18 S-IV).

Eligible participants were patients with inoperable CTEPH or persistent/recurrent CTEPH after PEA who had started a new riociguat therapy about 1 year before enrolment to the registry. CTEPH diagnosis was based on clinical, laboratory and imaging assessments in conformity with standard guidelines. Inoperability status had been established by an interdisciplinary team consisting of a PEA surgeon, a cardiac anesthesiologist, and a pulmonary hypertension specialist experienced in pharmacotherapy and the BPA procedure. Persistent/recurrent CTEPH was defined as increased pulmonary artery mean pressure $($ PAMP) $\geq 25 \mathrm{mmHg}$ concurrently with increased pulmonary vascular resistance $(\mathrm{PVR})>3$ Wood units (WU) evaluated during right heart catheterization at least 6 months after PEA. Riociguat indication and administration complied with applicable guidelines and the approved Adempas ${ }^{\circledR}$ Summary of Product Characteristics (SPC) with an initial dose of $1 \mathrm{mg}$ three times daily (tid) gradually titrated up to a maximum tolerated dose of $2.5 \mathrm{mg}$ tid. Continuation of dose up-titration was limited by the manifestation of side effects (most frequently symptomatic hypotension and dyspepsia) as required by SPC and standard of care.

The primary objective of the registry was to assess the impact of riociguat on the clinical parameters and quality of life in patients with inoperable CTEPH and patients with persistent/recurrent CTEPH after PEA in real-life settings.

Data were collected from patients' charts where information from routinely performed procedures and visits were recorded. For the purpose of the registry, the following data was collected: baseline characteristics including hemodynamic parameters at the time of riociguat initiation, riociguat dose, riociguat side effects, riociguat discontinuation if applicable, survival status, functional status by New York Heart Association (NYHA) class, a 6-minute walking distance (6MWD) test, Borg dyspnoea scale, and the EQ5D-5L questionnaire score filled in by each patient to evaluate their quality of life including

Table 1. Population Characteristics*.

\begin{tabular}{ll}
\hline Sex & $23(45.1 \%)$ \\
$\quad$ Males/ & $28(54.9 \%)$ \\
$\quad$ Females & $42(82.4 \%)$ \\
\hline CTEPH type & $9(17.6 \%)$ \\
$\quad$ Inoperable & \\
$\quad$ Residual after PEA & $36(70.6 \%)$ \\
\hline History of pulmonary embolism & $7(13.7 \%)$ \\
$\quad$ episode & \\
Repeated & $2(3.9 \%)$ \\
\hline NYHA functional class & $49(96.1 \%)$ \\
II & $67.7(64.4 ; 71.1) / 70.6(35.4 ; 87.1)$ \\
$\quad$ III & $28.0(26.4 ; 29.6) / 28.3(17.8 ; 46.1)$ \\
\hline Age at riociguat therapy initiation (years) & $328.6(296.5 ; 360.7) / 315.0(83.0 ; 520.0)$ \\
Body mass index - BMI $\left(\mathrm{kg} / \mathrm{m}^{2}\right)$ & $44.1(40.7 ; 47.6) / 42.0(21.0 ; 75.0)$ \\
Distance in 6MWD test $(\mathrm{m})$ & $9.2(8.3 ; 10.0) / 9.0(3.0 ; 17.0)$ \\
Pulmonary artery mean pressure - PAMP $(\mathrm{mmHg})$ & $2.4(2.3 ; 2.5) / 2.3(1.2 ; 4.1)$ \\
Pulmonary capillary wedge pressure - PCWP $(\mathrm{mmHg})$ & $8.1(7.2 ; 9.1) / 7.8(3.2 ; 16.0)$ \\
Cardiac index - CI $\left(\mathrm{L} / \mathrm{min} / \mathrm{m}^{2}\right)$ & $143.9(138.2 ; 149.6) / 145.0(97.0 ; 192.0)$ \\
Pulmonary arteriolar resistance - PAR $(\mathrm{WU})$ & $75.9(73.1 ; 78.6) / 76.0(53.0 ; 95.0)$ \\
Systemic blood pressure - systolic $(\mathrm{mmHg})$ & $75.3(71.2 ; 79.3) / 76.0(51.0 ; 119.0)$ \\
Systemic blood pressure - diastolic $(\mathrm{mmHg})$ & $367.4(231.2 ; 503.7) / 188.0(12.0 ; 2544)$ \\
Heart rate (beats per minute) &
\end{tabular}

\footnotetext{
*Number (\%) are presented for categorical variables, mean (95\% confidence interval)/ median (minimum; maximum) are presented for continuous variables.

CTEPH, thromboembolic pulmonary hypertension; PEA, pulmonary endarterectomy; NYHA, New York Heart Association; 6MWD, 6-minute walking distance; WU, Wood unit.
} 
Table 2. Change in endpoints from baseline to 5 months (mean 5.0 months; median 5.3 months) of riociguat treatment.

\begin{tabular}{|c|c|c|}
\hline Parameter & Change from baseline to Month $5 *$ & $P^{* *}$ \\
\hline \multicolumn{3}{|l|}{ Change of NYHA functional class, $n=37$} \\
\hline No change & $28(75.7 \%)$ & - \\
\hline Improvement by at least one class & $9(24.3 \%)$ & - \\
\hline Change of distance in 6MWD test $(\mathrm{m}), \mathrm{n}=22$ & /28.5 (-200.0; 220.0) & 0.066 \\
\hline Borg dyspnoea scale, $n=22$ & $/-0.5(-3.0 ; 4.0)$ & 0.366 \\
\hline Change of EQ5D-5L score, $n=22$ & $0.124(0.021 ; 0.226) / 0.101(-0.257 ; 0.866)$ & 0.020 \\
\hline Change of overall health status assessment (\%), $n=22$ & / $10.0(-20.0 ; 55.0)$ & 0.001 \\
\hline
\end{tabular}

*Number (\%) are presented for categorical variables, mean (95\% confidence interval)/median (minimum; maximum) are presented for continuous variables.

${ }^{* *} P$-value was calculated with the use of paired t-test comparing values from baseline and from the assessed time-point. Significant results are bold. NYHA, New York Heart Association; n, number of patients with data available for both assessed time-points; 6MWD, 6-minute walking distance.

Table 3. Change in endpoints from baseline to 1 year (mean 11.2 months; median 11.8 months) of riociguat treatment.

\begin{tabular}{|c|c|c|}
\hline Parameter & Change from baseline to Year $1^{*}$ & $P^{* *}$ \\
\hline \multicolumn{3}{|l|}{ Change of NYHA functional class, $n=28$} \\
\hline No change & $15(53.6 \%)$ & - \\
\hline Improvement by at least one class & $13(46.4 \%)$ & - \\
\hline Change of distance in $6 \mathrm{MWD}$ test $(\mathrm{m}), \mathrm{n}=17$ & /55.0 (-57.0; 242.0) & 0.006 \\
\hline Borg dyspnoea scale, $n=17$ & $/-1.0(-2.0 ; 3.0)$ & 0.177 \\
\hline Change of EQ5D-5L score, $n=18$ & $0.088(-0.063 ; 0.239) /-0.010(-0.375 ; 0.817)$ & 0.237 \\
\hline Change of overall health status assessment (\%), $\mathrm{n}=18$ & $/ 12.5(-30.0 ; 44.0)$ & 0.009 \\
\hline
\end{tabular}

* Number (\%) are presented for categorical variables, mean (95\% confidence interval)/ median (minimum; maximum) are presented for continuous variables.

** $P$-value was calculated with the use of paired t-test comparing values from baseline and from the assessed time-point. Significant results are bold. NYHA, New York Heart Association; n, number of patients with data available for both assessed time-points; 6MWD, 6-minute walking distance.

visual analogue scale (EQ-VAS) for a self-assessment of overall health status. The following time-points were considered for the final analysis: baseline data from the time of riociguat initiation, and from approximately 5 months and 1 year after the initiation of riociguat therapy.

The statistical analysis was performed in IBM SPSS Statistics 24. Continuous parameters in the analysis were described using the number of observations, mean, standard deviation, median, minimum, maximum and $95 \%$ confidence intervals (CI); discrete category parameters were described using absolute and relative frequency. To analyse registry objectives, the null hypothesis that there would be no difference in the values from baseline and the values from the selected time-point ( 5 or 12 months) for a particular clinical parameter was tested using the paired t-test at the 5\% significance level.

\section{RESULTS}

In total, 51 patients ( 23 males and 28 females) of a median age of 70.6 years with inoperable CTEPH or with persistent/recurrent CTEPH after PEA on riociguat therapy were enrolled for the retrospective collection of clinical parameters and quality of life data. Population characteristics are summarized in Table 1.

All patients used long-term anticoagulant therapy (warfarin in 45 patients, new oral anticoagulants in 6 patients). Before riociguat, 7 patients had received long- term therapy with subcutaneous treprostinil, 3 patients had received bosentan. For both previous treatments, patients had used stable doses more than 6 months before riociguat initiation.

The analysis of data collected at 5 months (mean 5.0 months; median 5.3 months) after riociguat initiation showed that $81.1 \%$ of patients received a maximal riociugat dose (2 $\mathrm{mg}$ and $2.5 \mathrm{mg}$ tid). Data from the start of therapy as well as from the 5-month visit were available in 37 patients for NYHA class, and in 22 patients for both the 6MWD test and the EQ5D-5L score. As described in Table 2, improvements from baseline to month 5 were observed in changes of distance measured in the 6MWD test (mean $35.3 \mathrm{~m}, 95 \% \mathrm{CI}: 2.6 ; 73.3 ; P=0.066$ ), changes of EQ5D-5L score (mean 0.124; 95\% CI: 0.021; 0.226; $P=0.020)$, and self-assessments of overall health status (mean 12.4\%; 95\% CI: 5.6; 19.2; $P=0.001$ ). NYHA class improved by at least one degree in $24.3 \%$ of patients. No change was observed using the Borg dyspnoea scale (mean -0.3 ; 95\% CI:-1.0; $0.4 ; P=0.366$ ).

The analysis of data collected approximately 1 year (mean 11.2 months; median 11.8 months) after riociguat initiation found that $85.8 \%$ of patients received a maximal riociguat dose ( $2 \mathrm{mg}$ and $2.5 \mathrm{mg}$ tid). Data from the start of therapy as well as from the 1-year visit were available in 28 patients for NYHA class, in 17 patients for the 6MWD test, and in 18 patients for the EQ5D-5L score. As described in Table 3, improvements from baseline to year 1 were observed in changes of distance measured in 
the 6MWD test (mean 57.2; 95\% CI: 19.1; 95.2; $P=0.006$ ), and self-assessments of overall health status (mean 12.6; 95\% CI: 3.7; 21.6; $P=0.009$ ). NYHA class improved by at least one degree in $46.4 \%$ of patients. No changes were observed using the Borg dyspnoea scale (mean $-0.5 ; 95 \%$ CI:-1.2; $0.2 ; P=0.177$ ) or EQ5D-5L score (mean 0.088, 95\% CI: $-0.063 ; 0.239 ; P=0.237)$.

Riociguat was well tolerated. In total, 4 patients reported side effects of riociguat treatment (gastrointestinal disorders, vomiting, symptomatic hypotension), and hospitalization was required in one case. Two patients discontinued treatment due to side effects.

The annual survival rate was $89.1 \%$ with $95 \% \mathrm{CI}$ : $70.0 \% ; 96.4 \%$.

\section{DISCUSSION}

Observational real-life studies in patients with inoperable CTEPH or persistent/recurrent pulmonary hypertension after PEA are important sources of data to complement randomized clinical trials, especially when enrolling patients with different clinical profiles.

The randomized, multi-centre, placebo-controlled clinical study CHEST-1 included patients with inoperable CTEPH and residual pulmonary hypertension after PEA: In patients receiving riociguat (nearly $90 \%$ of patients tolerated maximal doses of 2.0 and $2.5 \mathrm{mg}$ tid), significant improvements of distance in 6MWD was observed by 16 weeks. Distance lengthened by $39 \mathrm{~m}$ in the riociguat group and shortened by $6 \mathrm{~m}$ in the placebo group. Furthermore, pulmonary vascular resistance significantly decreased, levels of $\mathrm{N}$-terminal pro b-type natriuretic peptide (NP-proBNP) also decreased, and functional NYHA class improved in patients with riociguat. In an extension with open-label riociguat treatment for all participants, CHEST-2, improvement continued as assessed by the 6MWD test, and patients originally assigned to the placebo group achieved similar improvements as observed in the originally active group ${ }^{7}$.

The clinical profile of our study population was different from subjects of the pivotal CHEST-1 trial. Our patients were at higher risk: they were older (mean age of 67.7 years in comparison to 59 years in CHEST-1), with a higher proportion of males ( $45.1 \%$ vs. $34 \%$ in CHEST-1) and with more severe disease by functional class $(3.9 \%$ NYHA II and 96.1\% NYHA III vs. 32\% NYHA II and 64\% NYHA III in CHEST-1).

Despite the higher-risk population in our registry, treatment was well tolerated and we observed significant favourable impacts on clinical parameters. A high proportion of patients tolerated the two highest doses of riociguat ( $2 \mathrm{mg}$ and $2.5 \mathrm{mg}$ tid) on follow-up at (means) 5 and 11.2 months.

Mean changes of distance in 6MWD from baseline to months 5 and 11.2 in our study $(+35.3 \mathrm{~m}$ and $+57.2 \mathrm{~m}$ ) were similar to results obtained in the CHEST-1 and CHEST-2 studies. The Borg dyspnoea scale did not change significantly, but NYHA class improved in $24.3 \%$ of patients at month 5 and in $46.4 \%$ of patients at month
11.2. In comparison to CHEST-1, NYHA class had improved by at least one degree in $33 \%$ of patients and worsened in $5 \%$ of patients after 16 weeks of riociguat treatment.

Our results correspond with outcomes in the large EXPERT (EXPosurE Registry RiociguaT in patients with pulmonary hypertension) database of 956 patients with CTEPH receiving riociguat, of whom 419 patients were newly-treated patients. In one year, the mean change of distance in the 6MWD test was $+29 \mathrm{~m}$ and improvement by at least one NYHA class was observed in $26.2 \%$ of patients ${ }^{8}$. Similar results have been found in smaller comparable registries of patients with CTEPH receiving riociguat therapy ${ }^{9,10}$. Of note, NYHA class and 6MWD are important prognostic markers in CTEPH, and their improvement corresponds with the amelioration of survival in populations of treated patients ${ }^{11}$.

Quality of life in patients with untreated chronic pulmonary hypertension is poor, and is usually similar to quality of life in patients with advanced stages of pulmonary, cardiac or renal disorders ${ }^{12}$. Using the EQ5D-5L questionnaire for quality of life evaluation, we observed significant improvements both in EQ5D-5L score and overall self-assessed health status in comparison to values at therapy initiation after 5 months of riociguat treatment. At the follow-up at 11.2 months, improvements of overall self-assessed health status prevailed in comparison to the level reported at the time of treatment initiation. Amelioration of the EQ5D-5L score was also observed in the active group compared to the placebo in the CHEST1 study. In CHEST-2, improvements in quality of life were associated with improvements in the capacity to exercise ${ }^{13}$.

Annual survival rate $89.1 \%$ in our registry was lower than in the open-label follow-up of patients originally enrolled in CHEST-1 (97\%). However, it is important to stress the generally higher risk profile of patients in reallife settings.

One limitation of our registry is the small number of enrolled patients and especially the limited number of patients with data available for all time-points analysed. Another limitation is the absence of full range hemodynamic data as in the standard of care, it is not regularly assessed in all patients with stable disease. However, an assessment of clinical parameters clearly associated with patient prognosis (NYHA class, 6MWD test) is sufficient to evaluate the impacts of riociguat therapy in target populations.

\section{CONCLUSION}

Clinical registries enable data to be gained that is complementary to results of randomized clinical trials on the effectiveness and safety of treatment in real-life settings. This is also applicable to patients with inoperable CTEPH and persistent/recurrent pulmonary hypertension after PEA receiving riociguat. The available data on such patients shows, and the results of this non-interventional registry confirms, that riociguat therapy even in the realworld significantly improves functional NYHA class, dis- 
tance in the 6MWD test and quality of life. Treatment is well tolerated, and no new safety signal was identified in this retrospective analysis of data from a real-world patient population.

Acknowledgement: Supported by the by the research project Progres Q38/LF1. Funding for this research was provided by MSD s.r.o. Czech Republic. Medical writing and editorial assistance was provided by Jana Mašková of NEOX s.r.o, Pancířova 2, Prague. This assistance was also funded by MSD s.r.o. Czech Republic.

Author contributions: PJ: study design, data collection and interpretation, manuscript writing, final manuscript revision; DA, JK, VD: data collection and interpretation, manuscript writing, final manuscript revision; JL, AL: manuscript writing, final manuscript revision.

Conflict of interest statement: PJ has received fees and grants from Actelion Pharmaceuticals Ltd, AOP Orphan, and MSD. DA has received fees form AOP Orphan, and MSD. JL has received fees form MSD. The other authors have no conflict of interest.

\section{REFERENCES}

1. Kim NH, Delcroix M, Jais X, Madani MM, Matsubara H, Mayer E, Ogo T, Tapson VF, Ghofrani HA, Jenkins DP. Chronic thromboembolic pulmonary hypertension. Eur Respir J 2019;53(1):1801915.

2. Gall H, Hoeper MM, Richter MJ, Cacheris W, Hinzmann B, Mayer E. An epidemiological analysis of the burden of chronic thromboembolic pulmonary hypertension in the USA, Europe and Japan. Eur Respir Rev 2017;26(143).pii: 160121.

3. Hsieh WC, Jansa P, Huang WC, Nižnanský M, Omara M, Lindner J. Residual pulmonary hypertension after pulmonary endarterectomy. A meta-analysis. J Thorac Cardiovasc Surg 2018;156(3):1275-87.

4. Galiè N, Humbert M, Vachiery JL, Gibbs S, Lang I, Torbicki A, Simonneau G, Peacock A, Vonk Noordegraaf A, Beghetti M, Ghofrani A, Gomez Sanchez MA, Hansmann G, Klepetko W, Lancellotti P, Matucci M, McDonagh T, Pierard LA, Trindade PT, Zompatori M, Hoeper M. 2015 ESC/ERS Guidelines for the diagnosis and treatment of pulmonary hypertension: The Joint Task Force for the Diagnosis and Treatment of Pulmonary Hypertension of the European Society of Cardiology (ESC) and the European Respiratory Society (ERS) Endorsed by: Association for European Paediatric and Congenital Cardiology (AEPC), International Society for Heart and Lung Transplantation (ISHLT). Eur Respir J 2015;46(4):903-75.

5. Stasch JP, Pacher P, Evgenov OV. Soluble guanylate cyclase as an emerging therapeutic target in cardiopulmonary disease. Circulation 2011;123:2263-73.

6. Ghofrani HA, D'Armini AM, Grimminger F, Hoeper MM, Jansa P, Kim $\mathrm{NH}$, Mayer E, Simonneau G, Wilkins MR, Fritsch A, Neuser D, Weimann G, Wang C; CHEST-1 Study Group. Riociguat for the treatment of chronic thromboembolic pulmonary hypertension. N Engl J Med 2013;369:319-29.

7. Simonneau G, D'Armini AM, Ghofrani HA, Grimminger F, Hoeper MM, Jansa P, Kim NH, Wang C, Wilkins MR, Fritsch A, Davie N, Colorado $\mathrm{P}$, Mayer E. Riociguat for the treatment of chronic thromboembolic pulmonary hypertension: a long-term extension study (CHEST-2). Eur Respir J 2015;45:1293-1302.

8. Ghofrani HA, Gall H, Grünig E, Klose H, Halank M, Langleben D, Snijder R, Escribano P, Mielniczuk LM, Lange T, Vachiery JL, Wirtz H, Helmersen DS, Tsangaris I, Barberá JA, Pepke-Zaba J, Boonstra A, Rosenkranz S, Ulrich S, Steringer-Mascherbauer R, Gomez Sanchez M, Humbert M, Pittrow D, Simonneau G, Klotsche J, Williams E, Meier C, Hoeper M. Safety of Riociguat for the Treatment of Chronic Thromboembolic Pulmonary Hypertension: Final Data Cut from the EXPERT Registry, Am J Respir Crit Care Med 2019;199:A6067.

9. Halank M, Hoeper MM, Ghofrani HA, Meyer FJ, Stähler G, Behr J, Ewert R, Fletcher M, Colorado P, Nikkho S, Grimminger F. Riociguat for pulmonary arterial hy- pertension and chronic thromboembolic pulmonary hypertension: results from a phase II long-term extension study. Respir Med 2017;128:50-6.

10. Thor MC, Klooster L, Snijder RJ, Post MC, Mager JJ. Long-term clinical value and outcome of riociguat in chronic thromboembolic pulmonary hypertension. Int J Cardiol Heart Vasc 2019;22:163-8. doi: 10.1016/j.ijcha.2019.02.004

11. Delcroix M, Staehler G, Gall H, Grünig E, Held M, Halank M, Klose H, Vonk-Noordegraaf A, Rosenkranz S, Pepke-Zaba J, Opitz CF, Gibbs JSR, Lange TJ, Tsangaris I, Huscher D, Pittrow D, Olsson KM, Hoeper MM. Risk assessment in medically treated chronic thromboembolic pulmonary hypertension patients. Eur Respir J 2018;52:1800248.

12. Reis A, Santos M, Vicente M, Furtado I, Cruz C, Melo A, Carvalho L, Gonçalves F, Sa-Couto P, Almeida L. Health-related quality of life in pulmonary hypertension and its clinical correlates: A cross-sectional study. Biomed Res Int 2018;2018:3924517.

13. Mathai SC, Ghofrani HA, Mayer E, Pepke-Zaba J, Nikkho S, Simonneau G. Quality of life in patients with chronic thromboembolic pulmonary hypertension. Eur Respir J 2016;48(2):526-37. 